\title{
COLABORAÇÃO COM CLIENTES E INOVAÇÃO ABERTA NO DESENVOLVIMENTO ÀGIL DE SOFTWARE: UMA REVISÃO SISTEMÁTICA DE LITERATURA
}

\author{
COLLABORATION WITH CUSTOMERS AND OPEN INNOVATION IN THE AGILE \\ DEVELOPMENT OF SOFTWARE: A SYSTEMATIC LITERATURE REVIEW
}

Taiana Beatriz Junkes da Silveira Mestre em Administração Universidade do Estado de Santa Catarina - UDESC/ESAG. Florianópolis, Santa Catarina - Brasil. taijunkes@gmail.com

\section{Resumo}

Dannyela da Cunha Lemos Doutora em Administração Universidade do Estado de Santa Catarina - UDESC/ESAG. Florianópolis, Santa Catarina - Brasil. lemosda@gmail.com

Esta pesquisa investiga as interfaces entre os temas colaboração com clientes, inovação aberta, desenvolvimento tecnológico e desenvolvimento ágil na literatura científica internacional. Foi realizada uma revisão sistemática de literatura e bibliometria de artigos disponíveis nas bases EBSCO, Scopus e Web of Science, publicados entre o período de 2014-2019. Foi selecionado um portfólio de 58 artigos para análise. Constatou-se que a maioria das pesquisas tem estudado a colaboração com clientes no contexto do desenvolvimento de bens/produtos. De um total de 58 artigos que abordaram a colaboração com clientes identificou-se 31 no contexto de desenvolvimento de novos bens/produtos, 15 no de software, 3 no de bens/produtos e serviços e 1 no de bens/produtos e software. No que diz respeito ao desenvolvimento de software, a colaboração com clientes tem sido mais explorada dentro do tema metodologias ágeis. Foram identificados que 13 artigos aplicados a desenvolvimento de software têm como tema as metodologias ágeis.

Palavras-chave: Colaboração com clientes. Cooperação. Metodologias ágeis. Inovação aberta. Desenvolvimento de novos produtos.

\begin{abstract}
This research investigates the interfaces between the themes customer collaboration, open innovation, technological development and agile development in the international scientific literature. A systematic review of articles available in EBSCO, Scopus and Web of Science bases, published between 2014-2019, was carried out. After applying filters, a portfolio of 58 articles related to the theme was found, which formed the basis for a bibliometric research. It has been found that most research has studied collaboration with clients in the context of goods/ product development. From 58 articles that addressed collaboration with customers, 31 were identified in the context of the development of new goods/products, 15 in the software, 3 in the goods/products and services and 1 in the goods/products and software. With regard to software development, collaboration with clients has been further explored associated with agile methodologies theme. It has been identified 13 articles applied to software development with the theme of agile methodologies.
\end{abstract}


Key-words: Customer collaboration. Cooperation. Agile methodology. Open innovation. New product development.

\section{Cite como - American Psychological Association (APA)}

Silveira, T. B. J., \& Lemos, D. da C. (2021, Ed. Esp. jan./abr.). Colaboração com clientes e inovação aberta no desenvolvimento àgil de software: uma revisão sistemática de literatura. Revista de Gestao e Projetos (GeP), 12(1), 39-69. https://doi.org/10.5585/gep.v12i1.18645.

Agradecimento: Os autores agradecem o apoio institucional da FAPESC ao grupo de pesquisa Strategos da UDESC/ESAG."

\section{Introdução}

Com clientes cada vez mais exigentes e um mercado altamente competitivo, as empresas são desafiadas a inovar constantemente para se manterem e alcançarem os resultados esperados. A habilidade de estabelecer relações, identificar oportunidades e tirar proveito delas move a inovação, que consiste na abertura de novos mercados ou em novas formas de servir aquele existente (Tidd \& Bessant, 2015).

As empresas buscam cooperar para suprir a falta de recursos internos; ampliar a base de conhecimento, buscando conhecimento científico e tecnológico não disponível internamente; prover conhecimento sobre as necessidades e comportamentos do usuário; promover a aprendizagem compartilhada; reduzir os custos de pesquisa, desenvolvimento e de entrada no mercado; reduzir os riscos tecnológicos e mercadológicos; e reduzir tempo de desenvolvimento e, consequentemente, de retorno dos investimentos em inovação (Tether, 2002; Tidd, Bessant \& Pavitt, 2008).
Dentre as formas de colaboração praticadas entre as empresas, aquela realizada junto aos clientes no processo de desenvolvimento de produtos tem se tornado fundamental. De acordo com Harhoff, Mueller e Van Reenen (2014), os clientes são o parceiro de cooperação mais importante para as empresas. E a principal contribuição para inovação em produtos vem deles segundo Gómez, Salazar e Vargas (2016).

Tal questão se torna especialmente importante no contexto das empresas de software, que possuem o desafio de equilibrar as expectativas do consumidor, entregando os novos produtos e valor mais rápido do que nunca, para se manterem competitivas frente aos seus concorrentes (Sommer, Hedegaard, Dukovska-Popovska \& Steger-Jensen, 2015). Nesse sentido, a necessidade de aproximação com os clientes tornou-se um imperativo nesse tipo de indústria, cujo ciclo de vida dos produtos e a necessidade de implementação de novas versões de software precisam satisfazer constantemente suas necessidades.

No entanto, a compreensão dessas relações de colaboração, especialmente com 
clientes, ainda permanece pouco explorada na literatura científica internacional. Füller, Mühlbacher, Matzler e Jawecki (2009) constataram que existe pouca pesquisa sobre as experiências dos consumidores nos processos de desenvolvimento de novos produtos, apesar de haver novas formas de colaboração entre produtor e consumidor, viabilizadas pela tecnologia da informação. Na mesma linha, Bergadano, Bosio e Spagnolo classificam como escassa a literatura científica sobre a colaboração com clientes no desenvolvimento de software. Em complemento, em estudo bibliométrico sobre inovação aberta, Randhawa Wilden e Hohberger (2016) destacam a pouca atenção dada ao papel do usuário como fonte externa para inovação.

Em leitura exploratória sobre o tema, verificou-se que a colaboração com clientes costuma ser abordada nas discussões acerca de inovação aberta (Chesbrough, 2003) e desenvolvimento tecnológico ou de produto (Cooper, 2006). Dessa forma, procedeu-se uma revisão sistemática de literatura e bibliometria com base em artigos encontrados nas bases EBSCO, Scopus e Web of Science, publicados entre o período de 2014-2019, utilizando-se como base os descritores: colaboração com clientes, inovação aberta e desenvolvimento tecnológico.

No aprofundamento das leituras, observou-se no estudo da literatura que aborda cooperação com clientes para inovação um foco essencialmente centrado no contexto do desenvolvimento de bens/produtos como, por exemplo, os estudos de Parida, Sjodin, Lenka e Wincent (2015), Bengtsson et al. (2015),

Gama, Sjödin e Frishammar (2017), Leber, Ivanišević, Borocki, Radišić e Ślusarczyk (2018), Cooper e Sommer (2018) e Cooper (2019).

Quando se trata de desenvolvimento de software, contudo, existe um outro conjunto de estudos, dentro da temática das metodologias ágeis que também aborda a colaboração com clientes, como por exemplo, os estudos de Ashmore e Wedlake (2016), Uikey e Suman, (2016), Otaduy e Diaz (2017) e Shim e Lee (2019). Para ampliar essa perspectiva, procedeu-se uma segunda rodada da revisão sistemática de literatura e bibliometria, no mesmo período de tempo e nas mesmas bases de dados da busca anterior, cruzando-se os descritores colaboração e metodologias ágeis. $\mathrm{O}$ conjunto das duas buscas resultou num portfólio final de 58 artigos.

Nesse sentido, ao buscar-se uma aproximação entre esses diferentes corpos de conhecimento, pode-se traçar um panorama mais amplo e completo acerca da temática. Portanto, este estudo tem como objetivo investigar as interfaces entre os temas colaboração com clientes, inovação aberta, desenvolvimento tecnológico e desenvolvimento ágil na literatura científica internacional.

A estrutura do presente artigo foi organizada com uma breve fundamentação teórica sobre o tema em questão. Em seguida, 
são apresentados os procedimentos metodológicos, a descrição e análise dos dados, bem como as considerações finais e, finalmente as referências bibliográficas.

\section{Fundamentação teórica}

De acordo com Cooper (2006) desenvolvimento tecnológico refere-se a projetos cujo resultado envolve a entrega de um novo conhecimento, nova tecnologia, capacidade técnica ou plataforma tecnológica e normalmente conduzem a projetos comerciais, como o desenvolvimento de novos produtos ou processos. O desenvolvimento tecnológico resulta de capacidades tecnológicas, que são habilidades técnicas, gerenciais e institucionais das empresas, resultante das habilidades acumuladas por seus membros ao longo do tempo e que as permitem utilizar seus equipamentos e informações de forma eficiente (Lall, 1993).

O desenvolvimento tecnológico tem sido cada vez mais impulsionado pela colaboração com parceiros externos à empresa, dentre os quais destacam-se basicamente dois tipos de parceria: Market-based partners (clientes e fornecedores) e Science-based partners (universidades e institutos de pesquisa) (Gama, Sjodin \& Frishammar, 2017). A inovação tecnológica bem sucedida exige formas complexas de organização empresarial. As organizações inovadoras devem formar ligações, a montante e a jusante, lateral e horizontal, pois sistemas tecnológicos avançados não são e não podem ser criados de forma isolada (Teece, 1992).

Frente à complexidade científica, a convergência tecnológica e aos altos custos das atividades de pesquisa, a cooperação em P\&D tem se tornado uma tendência mundial. Estas alianças possibilitam o complemento das competências empresariais, bem como a divisão de custos e riscos atrelados a inovação (Tigre, 2006). Para Piller e Walcher (2006), ao incorporar uma variedade muito maior de ideias e conhecimentos ao desenvolvimento de novos produtos, o desempenho desse processo deve melhorar e os produtos resultantes devem se adequar melhor às exigências do mercado. Deste modo, a cooperação com os externos é essencial para aumentar a capacidade de inovação (Enkel, Gassmann, \& Chesbrough, 2009).

No que tange às atividades colaborativas, recentemente, o paradigma mais comum das empresas inovadoras mudou de uma estratégia de inovação fechada para uma estratégia de "inovação aberta" (Park \& Kwon, 2018). Segundo Chesbrough (2003) a inovação fechada é caracterizada pelas empresas que geram suas próprias ideias de inovação, desenvolvem, constroem, comercializam, distribuem, prestam serviços, financiam e apoiam elas mesmas. As empresas que adotam esta estratégia de inovação entendem que o conhecimento está dentro de sua estrutura organizacional. A inovação aberta (open innovation, ou OI) é a ação conjunta de diferentes fontes, que conciliam esforços 
internos e externos à empresa, no processo de inovação, para além de realizar pesquisa e desenvolvimento, formar redes de colaboração de ideias e patentes voltadas ao desenvolvimento de inovações (Chesbrough, 2003).

As empresas que adotam a inovação aberta convidam o cliente para o processo de inovação como parceiro e coprodutor (Chesbrough, 2003). Para Chang e Taylor (2016) o envolvimento dos clientes nas fases de ideação e lançamento de novos produtos desenvolvidos melhora o desempenho financeiro e o tempo para comercialização deles, por meio da aceleração do time to market, enquanto a sua participação na fase de desenvolvimento desacelera este tempo de colocação no mercado, deteriorando a performance financeira do novo produto.

$$
\text { Com relação ao papel dos }
$$
clientes/usuários, Chesbrough (2003) destaca que eles têm informações importantes que podem ser vitais para a inovação aberta. Aqueles mais avançados e mais exigentes são inovadores e geralmente levam os produtos e serviços ao extremo, tentando fazer novas combinações. Para Von Hippel (2005), "usuários líderes" são usuários inovadores, que estão à frente da maioria dos usuários no que diz respeito a uma tendência de mercado importante. E neste sentido, esperam ser beneficiados sobre a solução para as necessidades que encontraram. Os "usuários líderes", muitas vezes estão à frente do mercado em termos de necessidade de inovar (Tidd \& Bessant, 2015).

Os componentes essenciais para o sucesso de novos produtos são a compreensão completa das necessidades e desejos dos clientes, a situação competitiva e a natureza do mercado (Cooper, 2013, 2019). Assim, a abordagem de integração ativa de consumidores no processo de desenvolvimento de novos produtos por meio de um VOC (voice-of-the-customer) é importante (Leber, Ivanišević, Borocki, Radišić, \& Ślusarczyk, 2018). A incorporação adequada da opinião de clientes, da sua interação e participação, melhora o desempenho do desenvolvimento de novos produtos (Hsu, 2016), além de melhorar o entendimento do usuário e suas ideias (Rose, Jones \& Furneaux, 2016).

No processo de desenvolvimento de um produto, os clientes têm três diferentes papéis: co-criador, usuário e recurso. Estes papéis podem ser expressos em termos de conhecimento e em determinados estágios de colaboração (ideação, desenvolvimento e testes). Assim, é necessário que as organizações que querem obter benefícios no desenvolvimento conjunto de produtos com clientes, avaliem as condições de integração de conhecimento e considerem a implementação de diferentes abordagens de colaboração (Eslami \& Lakemond, 2016).

No que diz respeito à colaboração com clientes especificamente no contexto de desenvolvimento de software, destaca-se a publicação do Manifesto ágil (Beck et al., 
2001) e a emergência das metodologias ágeis.

As metodologias ágeis são um conjunto de métodos de desenvolvimento de software baseados no desenvolvimento iterativo e incremental (Kumar \& Bhatia, 2012). As metodologias ágeis de desenvolvimento mudaram a natureza, coordenação, colaboração e comunicação com os clientes em projetos de desenvolvimento de software (Prabhu, 2016).

Segundo George, Scheibe, Townsend e Mennecke (2018), essa abordagem de desenvolvimento existe para gerar melhorias de software, entregas mais rápidas e com maior qualidade. Desta forma, as metodologias ágeis compartilham os atributos incremental, cooperativo, simples e adaptativo. O atributo incremental refere-se a pequenas versões de software, com ciclos rápidos de desenvolvimento. $\mathrm{O}$ atributo cooperativo refere-se a uma interação próxima do cliente e do desenvolvedor. $\mathrm{O}$ atributo simples implica que o próprio método é fácil de aprender e modificar e que está suficientemente documentado. E, o atributo adaptativo referese à capacidade para fazer e reagir às mudanças de última hora (Heredia, Garcia-Guzman, Amescua-Seco, \& Velasco-Diego, 2014). Assim, a adoção das metodologias ágeis tem como resultado um sistema que é adaptável e flexível e prospera com a mudança, tendo como elemento central uma definição de produto em constante evolução, que surge através de um planejamento dinâmico e de curto prazo (Cooper \& Sommer, 2018).

A Figura 1 demonstra o ciclo de desenvolvimento de produtos com base em metodologias ágeis. Esse ciclo, chamado de Sprint, geralmente de 1 a 4 semanas, é composto pelas seguintes etapas: (i) coleta de requisitos (identificação de necessidades dos clientes/usuários) e construção de um backlog de produto (lista de necessidades identificadas com requisitos de negócios a serem implementados); (ii) desenvolvimento do produto; (iii) testes de aceitação dos clientes; (iv) revisão/feedback; e (v) implementação das alterações/ melhorias solicitadas pelos clientes (se identificadas necessidades de alteração ou melhorias, inicia-se um novo ciclo) (Otaduy \& Diaz, 2017). 


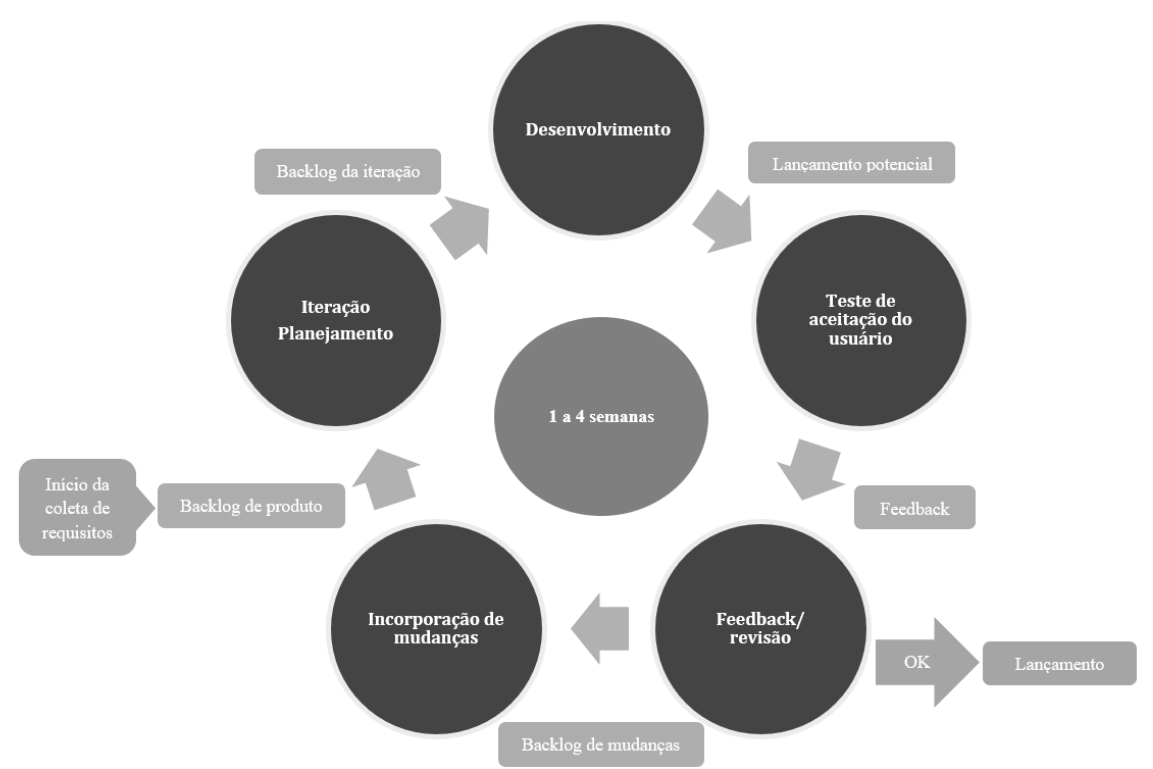

Figura 1. Ciclo de desenvolvimento ágil de software Fonte: Adaptado de Otaduy \& Diaz (2017).

De forma semelhante, Shim e Lee (2019) definiram esse processo de desenvolvimento, passando por cinco fases: $1^{\circ}$ ) Definição dos objetivos do projeto; $2^{\circ}$ ) Identificação do cliente; $3^{\circ}$ ) Entendimento do cliente; $4^{\circ}$ ) Entrega e validação da solução; $5^{\circ}$ ) Revisão da estratégia. Para eles, a $4^{\circ}$ etapa consiste na entrega (Delivery), com validação de hipóteses e entrega e validação da solução. As demais etapas consistem em processos relacionados a descoberta (Discovery), necessárias ao desenvolvimento de produtos. De forma complementar, Dingsøyr, Moe, Fægri, e Seim (2018) resumiram este processo a quatro fases: $1^{\circ}$ ) análise de necessidades; $2^{\circ}$ ) descrição da solução; $3^{\circ}$ ) construção; e $4^{\circ}$ ) aprovação.

Assim, o backlog existe desde o início do projeto e varia de acordo com o feedback dos clientes e os resultados das sprints, evoluindo ao longo do tempo à medida que o projeto avança e mais dados se tornam disponíveis, a partir dos experimentos e do feedback dos clientes (Cooper \& Sommer, 2018) para o status do projeto, dos requisitos e esclarecimentos de engenharia e testes. Para Kunda, Mulenga, Sinyinda e Chama (2018), as metodologias ágeis presumem o aumento dos laços de comunicação interna e entre as partes interessadas e são adequadas para projetos em que todas as partes mantêm uma relação de trabalho cordial. Caso contrário, usuários insatisfeitos podem frustrar os desenvolvedores durante o processo de desenvolvimento ou levar um bom produto ao desuso.

Um dos benefícios da iteração com o cliente/usuário final, no processo de 
desenvolvimento ágil de software, é a eliminação de atividades que não geram valor (de Oliveira Moraes, 2015). Além disso, a participação dos stakeholders através da concessão de feedback sob os projetos de desenvolvimento, diminui os custos de desenvolvimento, se comparados aos custos relacionados ao desenvolvimento de estudos de usuário, utilizados pela metodologia de desenvolvimento em cascata (Ashmore \& Wedlake, 2016).

\section{Procedimentos metodológicos}

A pesquisa em questão empregou primeiramente uma revisão sistemática da literatura sobre a temática colaboração com clientes. A revisão sistemática, segundo Demo (1995), é uma forma de pesquisa que utiliza a literatura sobre um determinado tema como fonte de dados. Para isto, são aplicados métodos explícitos e sistematizados de busca, realizando-se uma apreciação crítica e uma síntese sobre as informações selecionadas. A revisão sistemática de literatura permite dar sentido a grandes conjuntos de informações, mapeando áreas de incerteza e identificando áreas onde novos estudos são necessários (Petticrew \& Roberts, 2006).

Para realização da revisão sistemática de literatura tomou-se como base os três estágios sugeridos por Tranfield, Denyer e Smart (2003). O estágio 1 é o planejamento da revisão, envolvendo a identificação da necessidade, preparação da proposta e o desenvolvimento do protocolo de revisão. Já o estágio 2 refere-se à condução da revisão, com as etapas de identificação da pesquisa, seleção dos estudos, avaliação da qualidade, extração e síntese dos dados. O estágio 3 é o do relatório e disseminação, com a apresentação do relatório, recomendações e evidências práticas.

No que diz respeito ao planejamento da revisão, destaca-se que a motivação para a realização da busca sistemática deu-se pela necessidade de conhecer o status da produção científica internacional acerca da colaboração com clientes no contexto de empresas de software, considerando pesquisa empírica a ser realizada futuramente nessa área. A partir de leitura exploratória sobre o tema, inicialmente definiu-se como pergunta de pesquisa: o que tem sido pesquisado sobre colaboração com clientes e inovação aberta para o desenvolvimento de tecnologia?

Com base na pergunta de pesquisa e a partir dos termos-chave encontrados na leitura exploratória, elaborou-se o protocolo de revisão, que resultou numa primeira pesquisa no dia 30/03/2019, onde buscou-se pelos artigos que contivessem os termos (collaboration OR cooperation $O R$ partnership) AND customer AND "open innovation "AND "technology development"):

- na base da Scopus, a busca foi feita por todos os artigos dos últimos 5 anos, sem aplicar o filtro por artigos disponíveis;

- na base da EBSCO foram aplicados os filtros para busca dos textos completos disponíveis e somente artigos; e 
- na base Web of Science, ao replicar a busca pelos mesmos descritores não foram encontrados resultados. Então optou-se por fazer duas buscas separadas: a primeira com os termos (collaboration OR cooperation OR partnership) AND customer AND “open innovation"); e a segunda com os termos (collaboration OR cooperation OR partnership) AND customer AND “technology development”). Para estas duas pesquisas, a busca também foi feita por todos os artigos dos últimos 5 anos, sem aplicar o filtro por artigos disponíveis.

Ressalte-se que para o termo colaboração foram também incluídos os sinônimos cooperação e parceria, ambos combinados com o termo cliente, como forma de garantir maior abrangência nas buscas, uma vez que as leituras exploratórias revelaram o seu uso de forma intercambiável.

Após aprofundamento das leituras, percebeu-se que a literatura que aborda a cooperação com clientes para inovação tinha um foco essencialmente centrado no desenvolvimento de bens/produtos. Considerando que o interesse para a pesquisa deu-se no contexto de empresas de software, optou-se por realizar uma segunda busca, de forma complementar, incluindo-se nos descritores os termos desenvolvimento ágil e metodologias ágeis. Tal flexibilidade de pesquisa garante um protocolo que permite ao pesquisador a capacidade de ser criativo no processo, sem abrir mão de avaliações fidedignas (Tranfield, Denyer \& Smart, 2003).

Nesse sentido, a pergunta de pesquisa foi reformulada para: "o que tem sido pesquisado sobre colaboração com clientes, inovação aberta e metodologias ágeis para o desenvolvimento de tecnologia?". A partir disso trabalhou-se com os seguintes descritores: ((collaboration OR cooperation OR partnership) AND customer AND "open innovation "AND "technology development" e (("agile development" OR "agile methodology") AND customer), que foram pesquisados nas bases da EBSCO, Web of science e Scopus.

A segunda pesquisa, feita no dia $14 / 10 / 2019$, buscou os termos de pesquisa (("agile development" OR "agile methodology") AND customer), nas bases da Scopus, EBSCO e Web of Science, de todos os artigos dos últimos 5 anos, sem aplicar o filtro por artigos disponíveis. A Tabela 1 apresenta uma síntese da pesquisa realizada, por base de dados. 
Tabela 1. Pesquisa realizada por base de dados

\begin{tabular}{|c|c|c|c|c|}
\hline Busca & Base & Descritores & Data & $\begin{array}{c}\text { Resulta } \\
\text { dos }\end{array}$ \\
\hline 1 & Scopus & $\begin{array}{l}\text { ((collaboration OR cooperation OR partnership) AND customer AND "open } \\
\text { innovation "AND "technology development") }\end{array}$ & 30/03/2019 & 72 \\
\hline 2 & EBSCO & $\begin{array}{l}\text { ((collaboration OR cooperation OR partnership) AND customer AND "open } \\
\text { innovation "AND "technology development") }\end{array}$ & 30/03/2019 & 127 \\
\hline 3 & $\begin{array}{l}\text { Web of } \\
\text { science }\end{array}$ & $\begin{array}{l}\text { ((collaboration OR cooperation OR partnership) AND customer AND "open } \\
\text { innovation ") }\end{array}$ & 30/03/2019 & 53 \\
\hline 4 & $\begin{array}{l}\text { Web of } \\
\text { science }\end{array}$ & $\begin{array}{l}\text { ((collaboration OR cooperation OR partnership) AND customer AND } \\
\text { "technology development") }\end{array}$ & 30/03/2019 & 9 \\
\hline 5 & Scopus & (("agile development" OR "agile methodology") AND customer) & $14 / 10 / 2019$ & 48 \\
\hline 6 & EBSCO & (("agile development" OR "agile methodology") AND customer) & $14 / 10 / 2019$ & 17 \\
\hline 7 & $\begin{array}{l}\text { Web of } \\
\text { science }\end{array}$ & (("agile development" OR "agile methodology") AND customer) & $14 / 10 / 2019$ & 33 \\
\hline Total & & & & 359 \\
\hline
\end{tabular}

Fonte: Elaborado pelos autores, 2020.

O estágio 2 iniciou-se com o resultado da pesquisa que é uma lista completa dos artigos em que a revisão está baseada (Tranfield, Denyer \& Smart, 2003). Dessa forma, chegou-se a um portfólio total de 359 artigos, 144 deles correspondentes a base da EBSCO, 120 da base Scopus e 95 da base Web of Science. Para a seleção dos artigos foram aplicados filtros de:

- Exclusão de artigos duplicados (73);

- Exclusão de artigos com título alinhado em outra área da ciência (78);

- Exclusão de artigo com resumo e palavraschave não alinhados com a pesquisa (101); e
- Leitura sistemática e exclusão de artigos não alinhados com a pesquisa (49).

Os dados foram extraídos manualmente das bases de dados para planilha Excel. Na sequência foram organizados a partir de detalhes das fontes de informação (título, autoria, periódico e outros detalhes de publicação), além das características do estudo que permitiram identificar o alinhamento do tema com os objetivos da pesquisa (Tranfield, Denyer \& Smart, 2003). Finalmente chegou-se a um portfólio de análise de 58 artigos, cujas etapas estão sintetizadas na Figura 2.

$$
\text { No estágio } 3 \text { procedeu-se a }
$$
sistematização e análise dos dados dos 58 
Silveira, T. B. J., \& Lemos, D. da C. (2021, Ed. Esp. jan./abr.). Colaboração com clientes e inovação aberta no desenvolvimento àgil de software: uma revisão sistemática de literatura

artigos do portfólio que permitiu chegar ao relatório de pesquisa, cujos dados são apresentados no tópico resultados e discussão. Para análise do conteúdo dos artigos foram levados em consideração os seguintes tópicos: objetivos, população, abordagem, contexto, conceitos-chave, procedimentos metodológicos e resultados, ordenados de forma a facilitar a sumarização de cada estudo.

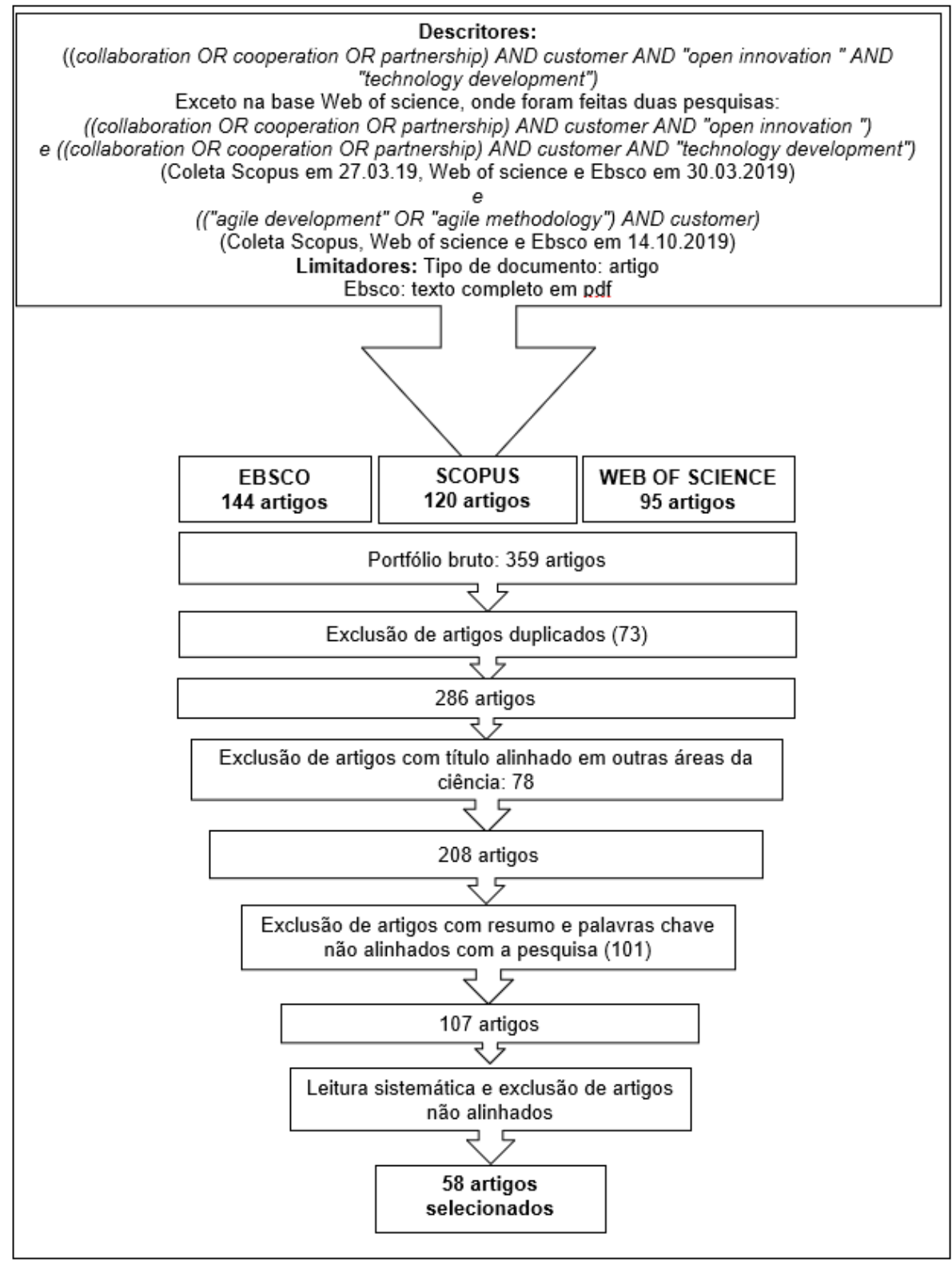

Figura 2. Etapas de seleção dos artigos na revisão sistemática de literatura

Fonte: Elaborado pelos autores, 2020. 
Como forma de obter-se uma visão geral dos dados levantados foi realizada a bibliometria. Para Maricato (2010) a bibliometria é o estudo de aspectos quantitativos relacionados a produção, disseminação e uso da informação registrada, desenvolvendo modelos e medidas matemáticas para elaborar previsões e ajudar na tomada de decisão. Como baseia-se em técnicas quantitativas, a bibliometria permite uma avaliação objetiva da produção científica (Araújo, 2006), e uma visão holística da temática a partir de um conjunto de parâmetros, como por exemplo o número total de citações de um autor, tópico, universidade ou país (Farrukh, Shahzad, Meng, Wu \& Raza, 2020).

Para realização da bibliometria considerou-se os seguintes tópicos: periódicos que mais trataram do tema e sua qualificação, a distribuição dos artigos por país e universidades em que foram publicados, a quantidade de artigos publicados nos últimos 5 anos, as palavras-chave que mais apareceram, as obras mais citadas e a frequência em que foram citadas nos artigos, a frequência de citação dos artigos analisados. Na sequência procedeu-se a categorização para identificar a medida de consenso entre os vários temas (Tranfield, Denyer \& Smart, 2003). Foram identificadas três grandes categorias: inovação aberta, colaboração com clientes e metodologias ágeis, bem como os pontos de interseção entre estas, nas quais os artigos do portfólio foram classificados.

\section{Resultados e discussão}

Este tópico apresenta dados gerais sobre os 58 artigos analisados, baseados na análise bibliométrica e discorre sobre os resultados da revisão sistemática de literatura. No que diz respeito à representatividade de artigos selecionados para esta pesquisa, a Figura 3 mostra os periódicos com a maior concentração de artigos. Estes periódicos comportaram $33 \%$ do total de artigos utilizados nesta pesquisa. 
Silveira, T. B. J., \& Lemos, D. da C. (2021, Ed. Esp. jan./abr.). Colaboração com clientes e inovação aberta no desenvolvimento àgil de software: uma revisão sistemática de literatura

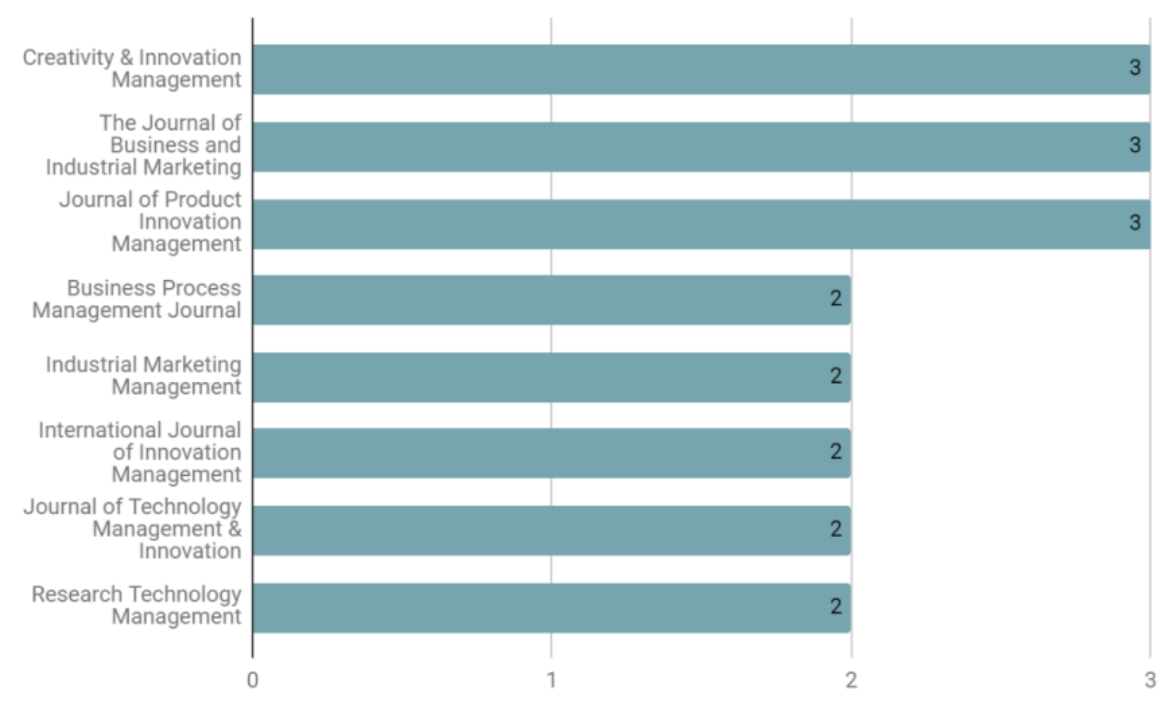

Figura 3. Periódicos mais representativos, a partir do número de artigos selecionados.

Fonte: Elaborado pelos autores, 2020.

Todos os periódicos tratam de assuntos relacionados à gestão da empresa. Destaca-se o escopo em marketing do The Journal of Business and Industrial Marketing e Industrial Marketing Management e o escopo em gestão e inovação de empresas do Creativity \& Innovation Management, International Journal of Innovation Management, Journal of Product Innovation Management, Business Process
Management Journal e Journal of Technology Management \& Innovation. Além disso, destacam-se algumas informações sobre estas revistas, conforme Tabela 2, foram avaliados o Qualis do quadriênio 2013-2016 e quatro destas revistas possuem Qualis A1, três tem Qualis A2 e um tem Qualis B2, o que atesta sua qualidade e relevância.

Tabela 2. Periódico, ISSN, Publisher, País e Qualis dos periódicos com mais artigos selecionados

\begin{tabular}{lcccc}
\hline \multicolumn{1}{c}{ Periódico } & ISSN & Publisher & País & Qualis \\
\hline Business Process Management Journal & $1463-7154$ & Emerald & Reino Unido & A1 \\
$\begin{array}{l}\text { Creativity \& Innovation Management } \\
\text { Industrial Marketing Management }\end{array}$ & $0963-1690$ & Wiley & Estados Unidos & A2 \\
& $0019-8501$ & Elsevier BV & Holanda & A1 \\
International Journal of Innovation Management & $1363-9196$ & World Scientific & Singapura & A2 \\
& & & & Estados Unidos \\
Journal of Product Innovation Management & $0737-6782$ & Wiley & Suécia & A2 \\
$\begin{array}{l}\text { Journal of Technology Management \& Innovation } \\
\text { Research Technology Management }\end{array}$ & $0718-2724$ & DOAJ & Reino Unido & B2 \\
The Journal of Business and Industrial Marketing & $0885-6308$ & Taylor \& Francis & Reino Unido & A1 \\
\hline
\end{tabular}

Fonte: Elaborado pelos autores, 2020.

Nota. Qualis pesquisado em https://sucupira.capes.gov.br/sucupira/public/consultas. 
Ao todo foram 25 países em todo o mundo que publicaram sobre o tema. A distribuição dos artigos por país de publicação demonstra que aqueles que mais publicaram artigos neste sentido foram a Suécia, com 9 publicações, seguida da Espanha e Coréia do Sul, com 7 e 4 artigos, respectivamente, conforme Figura 4.

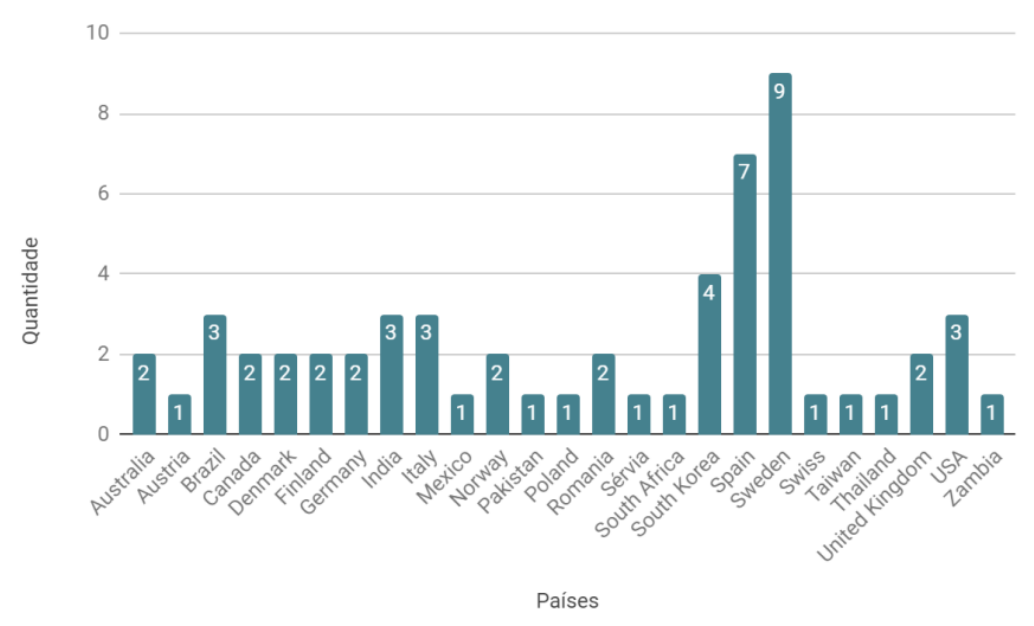

Figura 4. Frequência de artigos publicados por país.

Fonte: Elaborado pelos autores, 2020.

No que se refere à quantidade de artigos publicados nos últimos 5 anos, observa-se um equilíbrio nas publicações entre 2015 e 2018. Sendo que o ano que mais teve publicações sobre o tema foi 2016, com 14 artigos, seguido de 2015 (13 artigos), 2018 (12 artigos), 2017 (11 artigos). Os anos de 2014 e 2019, analisados parcialmente, tiveram 5 e 3 artigos respectivamente. Dentre as universidades que mais tem estudado o assunto destaca-se a Universidade de Tecnologia de Lulea, na Suíça, conforme mostra a Tabela 3. 
Silveira, T. B. J., \& Lemos, D. da C. (2021, Ed. Esp. jan./abr.). Colaboração com clientes e inovação aberta no desenvolvimento àgil de software: uma revisão sistemática de literatura

Tabela 3. Autores por universidades e países em que estão localizadas

\begin{tabular}{lcc}
\hline \multicolumn{1}{c}{ Universidade } & País & $\begin{array}{c}\text { Quantidade de autores por } \\
\text { universidade }\end{array}$ \\
\hline Luleå University of Technology & Suécia & 10 \\
Aachen University & Alemanha & 4 \\
Carlos III University of Madrid & Espanha & 4 \\
SINTEF & Noruega & 4 \\
Linköping University & Suécia & 4 \\
Iowa State University & Estados Unidos & 4 \\
Lucian Blaga University of Sibiu & Romênia & 4 \\
Aalborg University & Dinamarca & 3 \\
Universidad de La Rioja & Espanha & 3 \\
University of Girona & Espanha & 3 \\
Computer Science Lahore College for Women & Paquistão & 3 \\
University & & 3 \\
University of Vienna & Áustria & 3 \\
Ajou University & Coréia do Sul & 3 \\
University of Gävle & Suécia & 3 \\
Università di Torino & Itália & 3 \\
Aalto University & Finlândia & 3 \\
Penn State University & Canadá & 3 \\
\hline
\end{tabular}

Fonte: Elaborado pelos autores, 2020.

Dentre os 58 artigos selecionados, as cinco palavras-chave que mais se destacaram foram “Open Innovation", "Innovation”, "Agile development", "R\&D” e "SME”. É o que mostra a nuvem de palavras na Figura 5, a seguir. 


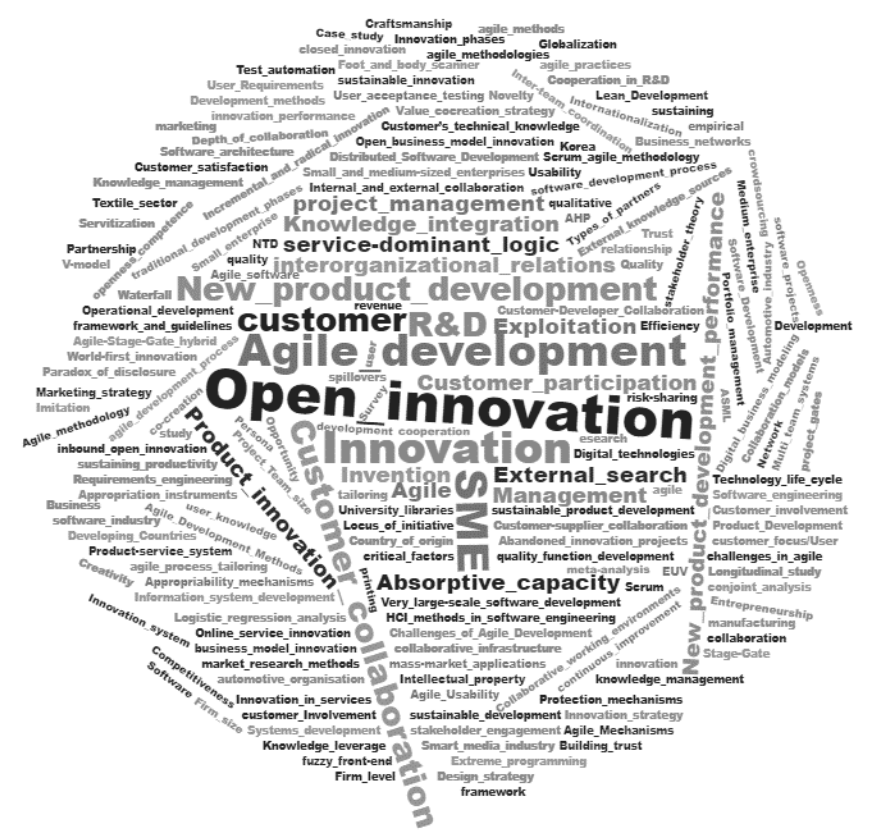

Figura 5. Palavras-chave mais citadas Fonte: Elaborado pelos autores, 2020.

Além disso, os 58 artigos selecionados possuem 3.280 referências. Dentre estas, as obras relacionadas à inovação aberta, colaboração com clientes e desenvolvimento ágil de software que mais apareceram foram as obras dos autores Laursen e Salter (2006), Chesbrough (2003), Beck et al. (2001), Van de Vrande, De Jong, Vanhaverbeke e De Rochemont (2009), Cohen e Levintahal (1990), entre outras. A seguir apresenta-se a Tabela 4 com dados das obras que foram referenciadas 5 vezes ou mais. 
Silveira, T. B. J., \& Lemos, D. da C. (2021, Ed. Esp. jan./abr.). Colaboração com clientes e inovação aberta no desenvolvimento àgil de software: uma revisão sistemática de literatura

Tabela 4. Obras mais citadas e frequência em que foram citadas nos artigos

\begin{tabular}{|c|c|c|c|c|}
\hline Autor(es) & Ano & Obra & Periódico/ Editora & Quantidade \\
\hline Laursen e Salter & 2006 & $\begin{array}{l}\text { Artigo: Open for innovation: the role of openness in } \\
\text { explaining innovation performance among U.K. } \\
\text { manufacturing firms. }\end{array}$ & Strateg Manag $J$ & 19 \\
\hline Chesbrough & 2003 & $\begin{array}{l}\text { Livro: Open innovation: The new imperative for } \\
\text { creating and profiting from technology. }\end{array}$ & $\begin{array}{l}\text { Harvard Business } \\
\text { Press. }\end{array}$ & 15 \\
\hline Beck et al & 2001 & Manifesto for Agile software development. & Agile Alliance & 12 \\
\hline $\begin{array}{l}\text { Van de Vrande, De Jong, Vanhaverbeke e } \\
\text { Rochemont. }\end{array}$ & 2009 & $\begin{array}{l}\text { Artigo: Open innovation in SMEs: trends, motives and } \\
\text { management challenges. }\end{array}$ & Technovation & 11 \\
\hline Cohen e Levinthal & 1990 & $\begin{array}{l}\text { Artigo: "Absorptive capacity: a new perspective on } \\
\text { learning and innovation" }\end{array}$ & $\begin{array}{l}\text { Administrative } \\
\text { Science Quarterly }\end{array}$ & 10 \\
\hline Dahlander e Gann & 2010 & Artigo: How Open Is Innovation? & Research Policy & 9 \\
\hline Enkel, Gassmann e Chesbrough & 2009 & $\begin{array}{l}\text { Artigo: Open } R \& D \text { and open innovation: Exploring } \\
\text { the phenomenon. }\end{array}$ & $R \& D$ Management & 9 \\
\hline Von Hippel & 1988 & Livro: The Sources of Innovation & $\begin{array}{l}\text { Oxford University } \\
\text { Press }\end{array}$ & 8 \\
\hline Huizingh & 2011 & $\begin{array}{l}\text { Artigo: Open innovation: State of the art and future } \\
\text { perspectives. }\end{array}$ & Technovation & 8 \\
\hline Tether & 2002 & $\begin{array}{l}\text { Artigo: Who co-operates for innovation, and why: An } \\
\text { empirical analysis. }\end{array}$ & Research Policy & 8 \\
\hline Leiponen e Helfat & 2009 & $\begin{array}{l}\text { Artigo: Innovation objectives, knowledge sources, and } \\
\text { the benefits of breadth }\end{array}$ & Strateg Manag & 7 \\
\hline Cassiman e Veugelers & 2002 & $\begin{array}{l}\text { Artigo: "R\&D Cooperation and Spillovers: Some } \\
\text { Empirical Evidence from Belgium" }\end{array}$ & $\begin{array}{l}\text { American Economic } \\
\text { Review }\end{array}$ & 7 \\
\hline Eisenhardt & 1989 & Building theories from case study research & $\begin{array}{l}\text { Academy of } \\
\text { Management } \\
\text { Review, }\end{array}$ & 6 \\
\hline Gassmann, Enkel e Chesbrough & 2010 & The future of open innovation & $R \& D$ Management & 6 \\
\hline Hair, Black, Babin e Anderson & 2006 & Multivariate data analysis (Vol. 6). & $\begin{array}{l}\text { NJ: Pearson } \\
\text { Prentice Hall. }\end{array}$ & 6 \\
\hline Lichtenthaler e Lichtenthaler & 2009 & $\begin{array}{l}\text { A capability-based framework for open innovation: } \\
\text { complementing absorptive capacity }\end{array}$ & $\begin{array}{l}\text { Journal of } \\
\text { Management Studies }\end{array}$ & 6 \\
\hline Parida, Westerberg e Frishammar & 2012 & $\begin{array}{l}\text { Inbound open innovation activities in high-tech SMEs: } \\
\text { The impact on innovation performance }\end{array}$ & $\begin{array}{l}\text { Journal of Small } \\
\text { Business } \\
\text { Management }\end{array}$ & 6 \\
\hline Prahalad e Ramaswamy & 2004 & $\begin{array}{l}\text { Co-creation experiences: the next practice in value } \\
\text { creation }\end{array}$ & $\begin{array}{l}\text { Journal of } \\
\text { Interactive } \\
\text { Marketing }\end{array}$ & 6 \\
\hline Von Hippel & 2009 & $\begin{array}{l}\text { Democratizing innovation: the evolving phenomenon } \\
\text { of user innovation }\end{array}$ & $\begin{array}{l}\text { International } \\
\text { Journal of } \\
\text { Innovation Science }\end{array}$ & 6 \\
\hline Zahra e George & 2002 & $\begin{array}{l}\text { Absorptive capacity: A review, reconceptualization, } \\
\text { and extension }\end{array}$ & $\begin{array}{l}\text { Academy of } \\
\text { Management Review }\end{array}$ & 6 \\
\hline Ahuja & 2000 & $\begin{array}{l}\text { Collaboration networks, structural holes, and } \\
\text { innovation: A longitudinal study }\end{array}$ & $\begin{array}{l}\text { Administrative } \\
\text { Science Quarterly }\end{array}$ & 5 \\
\hline Cooper & 2008 & $\begin{array}{l}\text { Perspective: The stage-gate idea-to-launch process- } \\
\text { Update, what's new, and next generation systems }\end{array}$ & $\begin{array}{l}\text { Journal of Product } \\
\text { Innovation } \\
\text { Management }\end{array}$ & 5 \\
\hline Faems, Van Looy e Debackere & 2005 & $\begin{array}{l}\text { Interorganizational collaboration and innovation: } \\
\text { Toward a portfolio approach }\end{array}$ & $\begin{array}{l}\text { Journal of Product } \\
\text { Innovation } \\
\text { Management }\end{array}$ & 5 \\
\hline Laursen e Salter & 2014 & $\begin{array}{l}\text { The Paradox of Openness: Appropriability External } \\
\text { Search and Collaboration }\end{array}$ & Research Policy & 5 \\
\hline
\end{tabular}

Fonte: Elaborado pelos autores, 2020. 
Os resultados da análise das obras mais citadas, demonstra uma forte presença do tema "capacidade de absorção" associado aos temas "colaboração com clientes" e "inovação aberta”. Também aparece entre os mais citados o Manifesto Ágil para o desenvolvimento ágil de software, demonstrando a sua importância no contexto das pesquisas sobre o desenvolvimento de produtos de software, além da associação com o tema "colaboração com clientes".

Por fim, dentre os 58 artigos selecionados para a pesquisa bibliométrica, 15 tiveram acima de 25 citações (de acordo com o Google Acadêmico), conforme Tabela 5.

Tabela 5. Artigos mais citados

\begin{tabular}{|c|c|c|c|c|c|}
\hline $\begin{array}{l}\mathbf{N}^{\circ} \text { de } \\
\text { citações }\end{array}$ & Ano & Autores & Título & Periódico & País \\
\hline 199 & 2016 & $\begin{array}{l}\text { Randhawa, Wilden e } \\
\text { Hohberger }\end{array}$ & $\begin{array}{l}\text { A Bibliometric Review of Open } \\
\text { Innovation: Setting a Research Agenda }\end{array}$ & $\begin{array}{l}\text { Journal of Product } \\
\text { Innovation } \\
\text { Management }\end{array}$ & Austrália \\
\hline 180 & 2016 & Chang e Taylor & $\begin{array}{l}\text { The Effectiveness of Customer } \\
\text { Participation in New Product } \\
\text { Development: A Meta-Analysis. }\end{array}$ & Journal of Marketing & $\begin{array}{l}\text { Estados } \\
\text { Unidos }\end{array}$ \\
\hline & & & $\begin{array}{l}\text { The Agile-Stage-Gate Hybrid Model: A } \\
\text { Promising New Approach and a New }\end{array}$ & $\begin{array}{l}\text { The Journal of } \\
\text { Product Innovation }\end{array}$ & \\
\hline 114 & 2016 & Cooper e Sommer & Research Opportunity & Management & Canadá \\
\hline 84 & 2015 & $\begin{array}{l}\text { Bengtsson, Lakemond, } \\
\text { Lazzarotti, Manzini, } \\
\text { Pellegrini e Tell }\end{array}$ & $\begin{array}{l}\text { Open to a Select Few? Matching Partners } \\
\text { and Knowledge Content for Open } \\
\text { Innovation Performance }\end{array}$ & $\begin{array}{l}\text { Creativity \& } \\
\text { Innovation } \\
\text { Management }\end{array}$ & Suécia \\
\hline 78 & 2018 & $\begin{array}{l}\text { Dingsøyr, Moe, Faegri, e } \\
\text { Seim }\end{array}$ & $\begin{array}{l}\text { Exploring software development at the } \\
\text { very large-scale: a revelatory case study } \\
\text { and research agenda for agile method } \\
\text { adaptation }\end{array}$ & $\begin{array}{l}\text { Empirical Software } \\
\text { Engineering }\end{array}$ & Noruega \\
\hline 72 & 2015 & $\begin{array}{l}\text { Parida, Sjödin Lenka, e } \\
\text { Wincent }\end{array}$ & $\begin{array}{l}\text { Developing Global Service Innovation } \\
\text { Capabilities }\end{array}$ & $\begin{array}{l}\text { Research Technology } \\
\text { Management }\end{array}$ & Suécia \\
\hline 41 & 2017 & $\begin{array}{l}\text { Goodman, Korsunova, e } \\
\text { Halme }\end{array}$ & $\begin{array}{l}\text { Our Collaborative Future: Activities and } \\
\text { Roles of Stakeholders in Sustainability- } \\
\text { Oriented Innovation }\end{array}$ & $\begin{array}{l}\text { Business Strategy and } \\
\text { the Environment }\end{array}$ & Finlândia \\
\hline 40 & 2015 & $\begin{array}{l}\text { Cruz-González, Lopez- } \\
\text { Saez e Navas-Lopez }\end{array}$ & $\begin{array}{l}\text { Absorbing knowledge from supply-chain, } \\
\text { industry and science: The distinct } \\
\text { moderating role of formal liaison devices } \\
\text { on new product development and novelty }\end{array}$ & $\begin{array}{l}\text { Industrial Marketing } \\
\text { Management }\end{array}$ & Espanha \\
\hline 39 & 2018 & Cooper e Sommer & $\begin{array}{l}\text { Agile-Stage-Gate for Manufacturers } \\
\text { Changing the Way New Products Are } \\
\text { Developed Integrating Agile project } \\
\text { management methods into a Stage-Gate } \\
\text { system offers both opportunities and } \\
\text { challenges }\end{array}$ & $\begin{array}{l}\text { Research Technology } \\
\text { Management }\end{array}$ & $\begin{array}{l}\text { Reino } \\
\text { Unido }\end{array}$ \\
\hline
\end{tabular}


Silveira, T. B. J., \& Lemos, D. da C. (2021, Ed. Esp. jan./abr.). Colaboração com clientes e inovação aberta no desenvolvimento àgil de software: uma revisão sistemática de literatura

Harhoff, Mueller e Van
$38 \quad 2014$ Reenen

$36 \quad 2015$ Law e Larusdottir

292016 Gómez, Salazar e Vargas

28

2016 Nordman e Tolstoy,

27

2017 Stefan e Bengtsson

262019 Cooper
What are the Channels for Technology Sourcing? Panel Data Evidence from German Companies

Whose Experience Do We Care About? Analysis of the Fitness of Scrum and Kanban to User Experience

Sources of Information as Determinants of Product and Process Innovation

The impact of opportunity connectedness on innovation in SMEs' foreign-market relationships

Unravelling appropriability mechanisms and openness depth effects on firm performance across stages in the innovation process

The drivers of success in new-product development Journal of Economics
\& Management
Strategy

Alemanha

International Journal of Human-Computer Reino Interaction Unido

PLoS ONE Espanha

Technovation Suécia

Technological

Forecasting and

Social Change

Suécia

Industrial Marketing

Management Canadá

Fonte: Elaborado pelos autores, 2020.

Nota. Quantidade de citações pesquisado em https https://scholar.google.com.br/scholar?q=, (em 11/12/2019).

A partir da sistematização dos dados foi possível classificar o portfólio dos 58 artigos em três grandes categorias: inovação aberta (5), colaboração com clientes (20) e metodologias ágeis (14). Alguns artigos foram classificados na interseção entre as categorias, da seguinte forma: inovação aberta e colaboração com clientes (14) e metodologias ágeis e colaboração com clientes (5), conforme demonstra a Figura 6. 


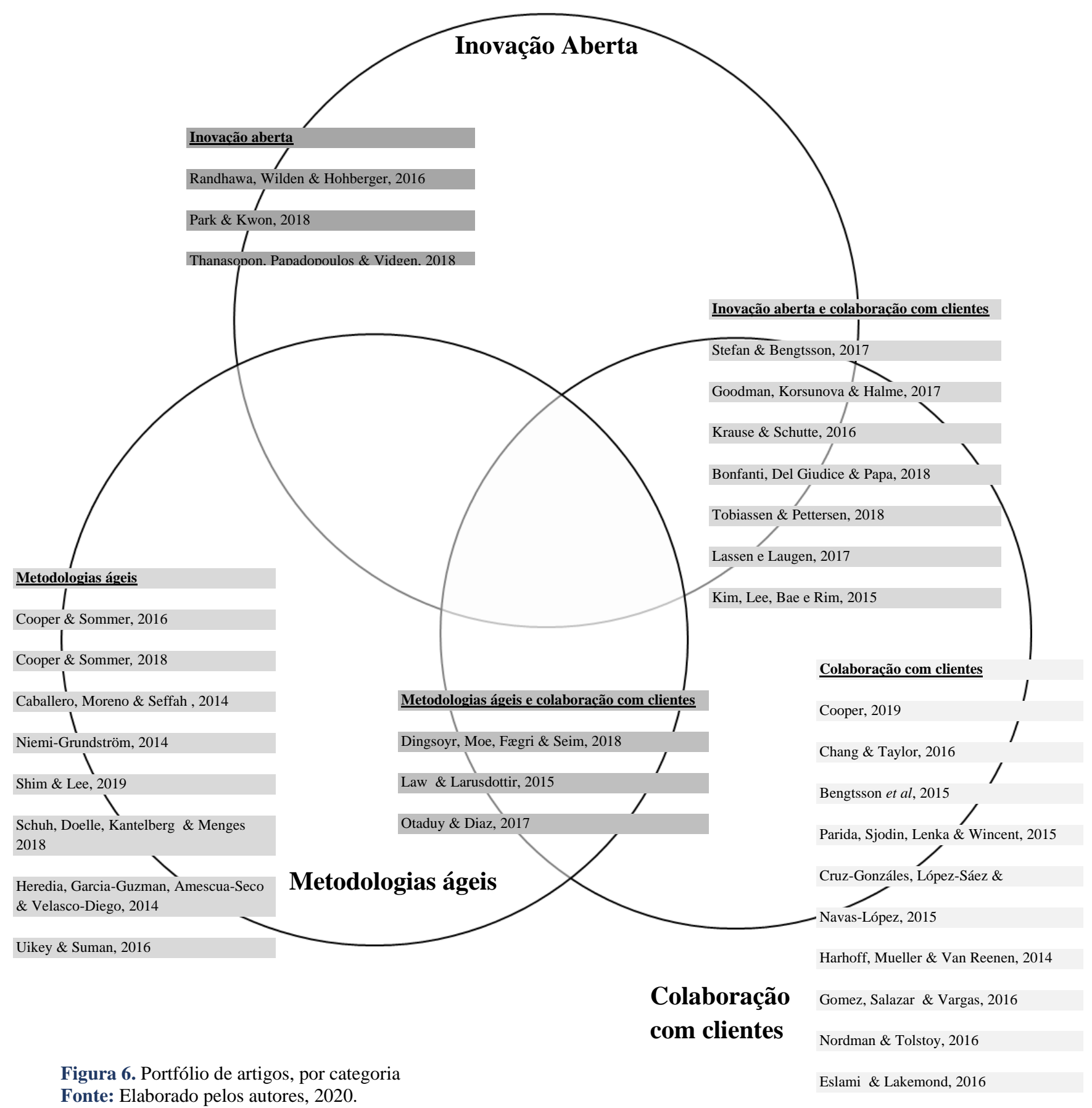

Fonte: Elaborado pelos autores, 2020.

dentre os 15 mais citados, e em 20 dentre os 58

É possível constatar que, para o contexto desta pesquisa, a categoria colaboração com clientes tem sido bastante pesquisada, uma vez que os resultados da análise do número de vezes em que o artigo foi citado demonstram que os artigos relacionados a este tema aparecem em maior volume (8) pesquisados.

Os oito artigos dentre os 15 mais citados classificados na categoria colaboração com clientes abordam a importância de diferentes fontes de informação (internas e externas) sobre inovações de produto e processo (Gómez, Salazar \& Vargas, 2016); a 
amplitude da participação de diferentes parceiros (Bengtsson et al., 2015); o seu conhecimento (Bengtsson et al., 2015; Parida, Sjodin, Lenka, \& Wincent, 2015); os mecanismos de ligação entre eles (CruzGonzáles, López-Sáez \& Navas-López, 2015); a cooperação entre parceiros de diferentes países (Harhoff, Mueller \& Van Reenen, 2014; Nordman \& Tolstoy, 2016); a participação dos clientes nas fases de ideação e lançamento do desenvolvimento de novos produtos (Chang \& Taylor, 2016); o desenvolvimento de insights no processo de desenvolvimento de novos produtos (Parida, Sjodin, Lenka \& Wincent, 2015); e direcionadores de sucesso para o desenvolvimento de um produto (Cooper, 2019), dentre outros aspectos relacionados a colaboração tanto com clientes como também com outros atores.

No conjunto dos demais artigos dessa categoria é relevante destacar os seguintes achados: a contribuição que o conhecimento do cliente tem no processo de inovação (Eslami \& Lakemond, 2016); a colaboração com os clientes é apoiada pelo desenvolvimento da capacidade absortiva (Ryzhkova \& Pesamaa, 2015); a adoção de perspectivas centrais da cocriação de valor tais como as opiniões do cliente, interação com o cliente e participação do cliente podem melhorar substancialmente o desempenho do desenvolvimento de produtos (Hsu, 2016); e o papel da participação dos clientes em processos de inovação realizados em pequenas e médias empresas (Rose, Jones \& Furneaux, 2016; Gama, Frishammar \& Parida, 2019).

Com relação à categoria inovação aberta, o artigo mais citado, de Randhawa, Wilden e Hohberger (2016), é uma pesquisa bibliométrica sobre a Inovação aberta (Open Innovation), que revelou três áreas distintas dentro da pesquisa de Open Innovation: (1) aspectos centrados na empresa, gerenciamento das redes de inovação aberta e (3) o papel dos usuários e comunidades na inovação aberta. Para Randhawa, Wilden e Hohberger (2016), até 2013 os estudos sobre Inovação aberta investigaram predominantemente aspectos centrados na empresa, com um foco particular no papel do conhecimento, tecnologia e P\&D, enquanto o gerenciamento das redes de inovação aberta e o papel dos usuários e comunidades na inovação aberta ficaram relativamente subpesquisados.

Os demais artigos reforçaram algumas questões relevantes no que diz respeito à relação firmada entre as organizações e os clientes para a inovação, tais como a importância de considerar as condições de cada empresa ao adotar uma estratégia de inovação aberta (Park \& Kwon, 2018); a experimentação do cliente como uma das atividades que promovem a inovação aberta na fase de front-end (Thanasopon, Papadopoulos \& Vidgen, 2018); os resultados do engajamento de clientes no processo de inovação da empresa (Rusu \& Avasilcai, 2015), e ainda a percepção dos clientes quanto 
ao seu papel dentro do paradigma de inovação aberta (Silva \& Pereira, 2015).

$\mathrm{Na}$ interseção entre as categorias inovação aberta e colaboração com clientes situam-se dentre os artigos mais citados os estudos de Goodman, Korsunova e Halme (2017) e Stefan e Bengtsson (2017) que abordaram as colaborações com diferentes atores dentro do contexto da inovação aberta, dentre os quais um deles é o cliente. Nessa categoria também pode-se destacar como resultados: a importância de uma abordagem de integração ativa de consumidores no processo de desenvolvimento de novos produtos (Leber, Ivanišević, Borocki, Radišić, \& Ślusarczyk, 2018) e as diferenças dos resultados da participação de clientes em projetos de inovação em diferentes partes do mundo (Tranekjer, 2017).

Na categoria de metodologias ágeis, os artigos de Dingsøyr, Moe, Fægri e Seim (2018) e Law e Larusdottir (2015) abordaram a aplicação das metodologias ágeis e o envolvimento do cliente no desenvolvimento de software. Além disso, pode-se destacar a questão dos testes de aceitação com a validação do software em ambiente real pelo público-alvo (Otaduy \& Diaz, 2017); a proposição de práticas e ferramentas para lidar com informações e comunicação no processo colaborativo (Bergadano, Bosio, \& Spagnolo, 2014) e a identificação de elementos críticos para a aplicabilidade da metodologia ágil Scrum em indústrias de software (Ali, Rehman, \& Anjum, 2017).
Já na interseção das categorias metodologias ágeis e colaboração com clientes destacam-se os artigos de Cooper e Sommer $(2016$; 2018) que abordaram a combinação dos métodos ágeis com o stage-gate (Cooper, 2008) e a sua aplicação em indústrias. Os autores têm estudado o assunto colaboração com clientes sob diferentes formas, abordando inclusive as metodologias ágeis de desenvolvimento. De uma maneira geral, os demais trabalhos nessa categoria destacam questões técnicas do desenvolvimento de software, sob a abordagem do desenvolvimento ágil, enfocando aspectos particulares das metodologias. Em todos os casos observa-se a ênfase no papel que o cliente desempenha no processo de desenvolvimento.

Observa-se que, de forma geral, pouco se tem estudado a respeito da colaboração com clientes no contexto de desenvolvimento de software. As pesquisas, em sua maioria, têm se concentrado no estudo da colaboração com clientes para o desenvolvimento de bens/produtos por indústrias. Dentre os artigos que subsidiaram esta pesquisa, destacam-se cinquenta (50), que abordaram a colaboração com clientes da seguinte forma: trinta e um (31) no contexto de desenvolvimento de novos bens/produtos, quinze (15) no de software, três (3) no de bens/produtos e serviços e um (1) no de bens/produtos e software. A colaboração com clientes no contexto de desenvolvimento de software tem sido mais explorada dentro do tema metodologias ágeis de desenvolvimento 
de software (13 artigos aplicados a

desenvolvimento de software têm como tema as metodologias ágeis).

As etapas de desenvolvimento de software podem ser resumidas em Discovery (Coleta de requisitos) e Delivery (Testes de aceitação/validação) (Dingsøyr, Moe, Fægri \& Seim, 2018; Otaduy \& Diaz, 2018; Shim \& Lee, 2019). Cada uma destas etapas tem diferentes objetivos e formas de participação dos clientes, conforme demonstram os achados deste artigo (Tabela 6).

Tabela 6. Participação dos clientes nas principais etapas de desenvolvimento ágil de software

\begin{tabular}{|c|c|c|}
\hline Etapa & Objetivo & Participação do cliente \\
\hline $\begin{array}{lll}\text { Discovery } & \text { (Coleta } & \text { de } \\
\text { requisitos) } & & \end{array}$ & $\begin{array}{l}\text { - Conhecer os clientes, entender as } \\
\text { necessidades dos clientes (Niemi- } \\
\text { Grundström, 2014); } \\
\text { - Entender as expectativas dos clientes e } \\
\text { introduzi-las nas especificações do } \\
\text { produto (Nicolaescu, Palade, Dumitrascu } \\
\text { \& Kifor, 2017); } \\
\text { - Definição das especificações completas, } \\
\text { precisas e formais (Uikey \& Suman, } \\
\text { 2016). }\end{array}$ & $\begin{array}{l}\text { - Validação de hipóteses por meio de } \\
\text { entrevistas, observações, prototipagem } \\
\text { e outras (Shim \& Lee, 2019); } \\
\text { - Reuniões frequentes para discussão do } \\
\text { status do projeto, requisitos, } \\
\text { esclarecimentos de engenharia e testes } \\
\text { (Nicolaescu, Palade, Dumitrascu, \& } \\
\text { Kifor, 2017). }\end{array}$ \\
\hline $\begin{array}{l}\text { Delivery (Desenvolvimento/ } \\
\text { Testes de } \\
\text { aceitação/validação) }\end{array}$ & $\begin{array}{l}\text { - Codificar os novos recursos e testá-los } \\
\text { (Otaduy \& Diaz, 2017); } \\
\text { - Validação do produto em um ambiente } \\
\text { real (Otaduy \& Diaz, 2017); } \\
\text { - Medir a satisfação do cliente, para } \\
\text { melhorar a qualidade das entregas } \\
\text { (Niemi-Grundström, 2014); } \\
\text { - Auxiliar na decisão por manter, alterar ou } \\
\text { descartar a solução (Shim \& Lee, 2019); } \\
\text { - Gerar uma nova lista de necessidades } \\
\text { (backlog) de clientes a serem } \\
\text { incorporadas ao backlog do produto } \\
\text { (Heredia, Garcia-Guzman, } \\
\text { Amescua-Seco, \& Velasco-Diego, 2014). }\end{array}$ & $\begin{array}{l}\text { - Validação do produto e fornecimento } \\
\text { de feedbacks (Otaduy \& Diaz, 2017; } \\
\text { Shim \& Lee, 2019); } \\
\text { - Reuniões frequentes para discussão do } \\
\text { status do projeto, requisitos, } \\
\text { esclarecimentos de engenharia e testes } \\
\text { (Nicolaescu, Palade, Dumitrascu, \& } \\
\text { Kifor, 2017). }\end{array}$ \\
\hline
\end{tabular}

Fonte: Elaborado pelos autores, 2020. 
Silveira, T. B. J., \& Lemos, D. da C. (2021, Ed. Esp. jan./abr.). Colaboração com clientes e inovação aberta no desenvolvimento àgil de software: uma revisão sistemática de literatura

Segundo de Oliveira Moraes, (2015), o desenvolvimento ágil e os princípios de suas metodologias são influenciados pelos conceitos de lean (enxuto), pois prezam por formas de eliminar desperdícios e atividades que não agregam valor. E, desta forma, o pensamento enxuto parte de uma profunda compreensão dos clientes e enfatiza que, ao desenvolver serviços e produtos, eles são o foco. Assim, conhecer as suas necessidades é vital, pois a qualidade do serviço depende de sua percepção (Niemi-Grundström, 2014). Para Bergadano, Bosio e Spagnolo (2014), no desenvolvimento de software, o cliente deve ter um papel colaborativo, representativo, autorizado, comprometido, além de conhecer a equipe de desenvolvimento. Ele deve compartilhar o mesmo trabalho com a equipe, trabalhando diariamente durante todo o projeto, de maneira próxima e se comunicando frequentemente com os membros.

Ashmore e Wedlake (2016), ao fazerem um estudo comparativo entre as metodologias ágeis de desenvolvimento de software e a abordagem tradicional, identificaram que os clientes preferem estar envolvidos em todo o processo de design e desenvolvimento de um produto, ao invés de aparecerem apenas no início (estudo de usabilidade) e no final (teste beta) do projeto. Assim, para Nicolaescu, Palade, Dumitrascu e Kifor (2017), o cliente deve sempre estar em um relacionamento próximo com a equipe de desenvolvimento, através de reuniões frequentes para discussão do status do projeto, dos requisitos e esclarecimentos de engenharia e testes.

Dentre os benefícios da iteração com o cliente/usuário final, no processo de desenvolvimento ágil de software, destacam-se a maior facilidade na compreensão dos comportamentos humanos (fatores que determinam o uso dos produtos, como as pessoas realmente utilizam e em que contexto, os fatores que determinam a adoção e aceitabilidade do produto por uma determinada comunidade, o que pode ser usado ou precisa ser melhorado na linha de comunicação entre desenvolvedores e usuários de um serviço, como o público-alvo se sente em relação a si mesmo no contexto do uso do produto e se eles parecem mais satisfeitos, inteligentes ou informados) (Caballero, Moreno, \& Seffah, 2014), a eliminação de atividades que não geram valor (de Oliveira Moraes, 2015) e a diminuição de custos de desenvolvimento (Ashmore \& Wedlake, 2016).

\section{Considerações finais}

Esta pesquisa teve como objetivo investigar as interfaces entre os temas colaboração com clientes, inovação aberta, desenvolvimento tecnológico e desenvolvimento ágil na literatura científica internacional. Para isto, foram selecionados 58 artigos, publicados nas bases de dados EBSCO, Scopus e Web of Science. Com base neles foi possível estabelecer um panorama geral sobre o tema colaboração com clientes, no contexto de empresas de software. 
No período avaliado, observou-se que 2016 foi o ano com mais estudos sobre o assunto. Além disso, o país que mais tem estudado o tema é a Suécia, seguida da Espanha e Coréia do Sul. Da mesma forma, a universidade que mais tem estudado o assunto é a Lulea University of Technology, na Suécia. As palavras-chave mais utilizadas foram "Open Innovation", "Innovation”, "Agile development", “R\&D” e "SME”.

No que diz respeito às referências dos artigos, as obras de Laursen e Salter (2006), Chesbrough (2003), Beck et al. (2001), Van de Vrande, De Jong, Vanhaverbeke e De Rochemont (2009) e Cohen e Levinthal (1990) se destacaram. E os temas mais citados indicam uma associação elevada do tema capacidade de absorção com "colaboração com clientes" e "inovação aberta" e ainda uma associação entre o tema "metodologias ágeis" e "colaboração com clientes".

A análise do portfólio dos 58 artigos permitiu classificá-los em três categorias temáticas, a saber: inovação aberta, colaboração com clientes e metodologias ágeis. A categoria colaboração com clientes apresentou o maior volume de resultados, sendo oito dentre os 15 mais citados e 20 dentre os 58 pesquisados. Também pode se identificar 14 artigos na interseção entre as categorias inovação aberta e colaboração com clientes, e 5 artigos na interseção entre as categorias metodologias ágeis e colaboração com clientes.
Desta forma, este artigo pode contribuir para estudos sobre o tema colaboração com clientes associado à inovação aberta e metodologias ágeis. No que concerne às limitações desta pesquisa, a amostra poderia ser de um período maior do que cinco anos. Isto possibilitaria uma visão mais ampla da movimentação acadêmica acerca do tema.

Para trabalhos futuros sugere-se a realização de estudos teóricos-empíricos que abordem a colaboração com clientes sob a perspectiva do desenvolvimento de software, uma vez que a pesquisa mostrou que são poucos os artigos que tratam da colaboração nessa vertente, bem como o papel das metodologias ágeis como impulsionadoras desse processo.

\section{Referências}

Ahuja, G. (2000). Collaboration networks, structural holes, and innovation: A longitudinal study. Administrative science quarterly, 45(3), 425-455.

Ali, A., Rehman, M., \& Anjum, M. (2017). Framework For Applicability Of Agile Scrum Methodology: A Perspective Of Software Industry. International journal of advanced computer science and applications, 8(9), 225-232.

Araújo, C. A. (2006). Bibliometria: evolução histórica e questões atuais. Em questão, 12(1), 11-32.

Ashmore, S., \& Wedlake, M. (2016). Developing the product your customer really wants: the value of an Agile partnership. Information Resources Management Journal (IRMJ), 29(3), 1-11. 
Badillo, E. R., Galera, F. L., \& Serrano, R. M. (2014). Cooperation in R\&D, firm size and type of partnership: Evidence for the Spanish automotive industry. Research Institute of Applied Economics Working Paper, 30, 1-32.

Banerjee, S., \& Sharma, A. K. (2015). Cocreation as a risk-sharing strategy for the development of innovative EUV lithography technology in the semiconductor industry. Technology Analysis \& Strategic Management, 27(9), 1097-1113.

Beck, K., Beedle, M., Van Bennekum, A., Cockburn, A., Cunningham, W., Fowler, M., ... \& Kern, J. (2001). Manifesto for agile software development.

Bengtsson, L., Lakemond, N., Lazzarotti, V., Manzini, R., Pellegrini, L., \& Tell, F. (2015). Open to a select few? Matching partners and knowledge content for open innovation performance. Creativity and innovation management, 24(1), 72-86.

Bergadano, F., Bosio, G., \& Spagnolo, S. (2014). Supporting collaboration between customers and developers: a framework for distributed, agile software development. International Journal of Distributed Systems and Technologies (IJDST), 5(2), 1-16.

Bonfanti, A., Del Giudice, M., \& Papa, A. (2018). Italian craft firms between digital manufacturing, open innovation, and servitization. Journal of the Knowledge Economy, 9(1), 136-149.

Brasseur, T. M., Mladenow, A., \& Strauss, C. (2017). Open business model innovation: Literature review and agenda for future research. Бизнес-информатика, (4 (42) eng).

Caballero, L., Moreno, A. M., \& Seffah, A. (2014, September). Persona as a tool to involving human in agile methods: contributions from hci and marketing. In International Conference on HumanCentred Software Engineering (pp. 283290). Springer, Berlin, Heidelberg.
Cassiman, B., \& Veugelers, R. (2002). R\&D cooperation and spillovers: some empirical evidence from Belgium. American Economic Review, 92(4), 1169-1184.

Chang, W., \& Taylor, S. A. (2016). The effectiveness of customer participation in new product development: A metaanalysis. Journal of Marketing, 80(1), 4764.

Chesbrough, H. W. (2003). Open innovation: The new imperative for creating and profiting from technology. Harvard Business Press.

Codini, A. P. (2015). Business networks along innovation life cycle. Journal of Business \& Industrial Marketing, 30(3-4), 329-341.

Cohen, W. M., \& Levinthal, D. A. (1990). Absorptive capacity: A new perspective on learning and innovation. Administrative science quarterly, 128-152.

Cooper, R. G. (2006). Managing technology development projects. Research-Technology Management, 49(6), 23-31.

Cooper, R. G. (2008). Perspective: The stage-gate ${ }^{\circledR}$ idea-to-launch processupdate, what's new, and nexgen systems. Journal of product innovation management, 25(3), 213-232.

Cooper, R. G. (2013). New products: What separates the winners from the losers and what drives success. PDMA handbook of new product development, 3-34.

Cooper, R. G., \& Sommer, A. F. (2016). The agile-stage-gate hybrid model: A promising new approach and a new research opportunity. Journal of Product Innovation Management, 33(5), 513-526.

Cooper, R. G., \& Sommer, A. F. (2018). Agile-Stage-Gate for Manufacturers: Changing the Way New Products Are Developed Integrating Agile project management methods into a Stage-Gate system offers both opportunities and 
challenges. Research-Technology

Management, 61(2), 17-26.

Cooper, R. G. (2019). The drivers of success in new-product development. Industrial Marketing Management, 76, 36-47.

Cruz-González, J., López-Sáez, P., \& NavasLópez, J. E. (2015). Absorbing knowledge from supply-chain, industry and science: The distinct moderating role of formal liaison devices on new product development and novelty. Industrial Marketing Management, 47, 75-85.

Dahlander, L., \& Gann, D. M. (2010). How open is innovation?. Research policy, 39(6), 699-709.

da Silva, F. M., Oliveira, E. A. D. A. Q., \& de Moraes, M. B. (2016). Innovation development process in small and medium technology-based companies. RAI Revista de Administração e Inovação, 13(3), 176189.

de Oliveira Moraes, R. (2015). Lean Development e os Métodos Ágeis de Desenvolvimento. Revista ESPACIOS Vol. $36\left(N^{o} 19\right)$ Año 2015.

Demo, P. (1995). Metodologia científica em ciências sociais. Editora Atlas.

Dingsøyr, T., Moe, N. B., Fægri, T. E., \& Seim, E. A. (2018). Exploring software development at the very large-scale: a revelatory case study and research agenda for agile method adaptation. Empirical Software Engineering, 23(1), 490-520.

Eisenhardt, K. M. (1989). Building theories from case study research. Academy of management review, 14(4), 532-550.

Enkel, E., Gassmann, O., \& Chesbrough, H. (2009). Open R\&D and open innovation: exploring the phenomenon. $R \& d$ Management, 39(4), 311-316.

Eslami, M. H., \& Lakemond, N. (2016). Knowledge integration with customers in collaborative product development projects. Journal of Business \& Industrial Marketing.

Faems, D., Van Looy, B., \& Debackere, K. (2005). Interorganizational collaboration and innovation: Toward a portfolio approach. Journal of product innovation management, 22(3), 238-250.

Farrukh, M., Shahzad, I. A., Meng, F., Wu, Y., \& Raza, A. (2020). Three decades of research in the technology analysis \& strategic management: a bibliometrics analysis. Technology Analysis \& Strategic Management, 1-17.

Füller, J., Mühlbacher, H., Matzler, K., \& Jawecki, G. (2009). Consumer empowerment through internet-based cocreation. Journal of management information systems, 26(3), 71-102.

Gama, F., Sjödin, D. R., \& Frishammar, J. (2017). Managing interorganizational technology development: Project management practices for market-and science-based partnerships. Creativity and Innovation Management, 26(2), 115-127.

Gama, F., Frishammar, J., \& Parida, V. (2019). Idea generation and open innovation in SMEs: When does market-based collaboration pay off most?. Creativity and Innovation Management, 28(1), 113-123.

Gassmann, O., Enkel, E., \& Chesbrough, H. (2010). The future of open innovation. $R \& d$ Management, 40(3), 213-221.

George, J. F., Scheibe, K., Townsend, A. M., $\&$ Mennecke, B. (2018). The amorphous nature of agile: no one size fits all. Journal of Systems and Information Technology.

Gómez, J., Salazar, I., \& Vargas, P. (2016). Sources of information as determinants of product and process innovation. PloS one, 11(4), e0152743.

Goodman, J., Korsunova, A., \& Halme, M. (2017). Our collaborative future: Activities and roles of stakeholders in sustainability-oriented innovation. Business 
Strategy and the Environment, 26(6), 731753.

Gruner, R. L., \& Power, D. (2017). What's in a crowd? Exploring crowdsourced versus traditional customer participation in the innovation process. Journal of Marketing Management, 33(13-14), 1060-1092.

Hair, J. F., Black, W. C., Babin, B. J., Anderson, R. E., \& Tatham, R. L. (2006). Multivariate data analysis (Vol. 6): Pearson Prentice Hall Upper Saddle River.

Harhoff, D., Mueller, E., \& Van Reenen, J. (2014). What are the channels for technology sourcing? Panel data evidence from German companies. Journal of Economics \& Management Strategy, 23(1), 204-224.

Heredia, A., Garcia-Guzman, J., Amescua-Seco, A., \& Velasco-Diego, M. (2014). Agile practices adapted to mass-market application development. Journal of Software: Evolution and Process, 26(9), 818-828.

Hochleitner, F. P., Arbussà, A., \& Coenders, G. (2016). World-first innovations in an open innovation context. Journal of technology management \& innovation, 11(3), 50-58.

Hofstetter, R., Aryobsei, S., \& Herrmann, A. (2018). Should you really produce what consumers like online? Empirical evidence for reciprocal voting in open innovation contests. Journal of Product Innovation Management, 35(2), 209-229.

Hsu, Y. (2016). A value cocreation strategy model for improving product development performance. Journal of Business \& Industrial Marketing.

Huizingh, E. K. (2011). Open innovation: State of the art and future perspectives. Technovation, 31(1), 2-9.

Kim, E., Lee, D., Bae, K., \& Rim, M. (2015). Developing and evaluating new ICT innovation system: case study of Korea's smart media industry. ETRI Journal, 37(5), 1044-1054.

Krause, W., \& Schutte, C. S. L. (2015). A perspective on open innovation in small-and medium-sized enterprises in South Africa, and design requirements for an open innovation approach. South African Journal of Industrial Engineering, 26(1), 163-178.

Kumar, G., \& Bhatia, P. K. (2012). Impact of agile methodology on software development process. International Journal of Computer Technology and Electronics Engineering (IJCTEE), 2(4), 46-50.

Kunda, D., Mulenga, M., Sinyinda, M., \& Chama, V. (2018). Challenges of Agile Development and Implementation in a Developing Country: A Zambia Case Study. J. Comput. Sci., 14(5), 585-600.

Lall, S. (1993). Understanding technology development. Development and change, 24(4), 719-753.

Lassen, A. H., \& Laugen, B. T. (2017). Open innovation: on the influence of internal and external collaboration on degree of newness. Business Process Management Journal.

Laursen, K., \& Salter, A. (2006). Open for innovation: the role of openness in explaining innovation performance among UK manufacturing firms. Strategic management journal, 27(2), 131-150.

Laursen, K., \& Salter, A. J. (2014). The paradox of openness: Appropriability, external search and collaboration. Research policy, 43(5), 867-878.

Law, E. L. C., \& Lárusdóttir, M. K. (2015). Whose experience do we care about? Analysis of the fitness of scrum and kanban to user experience. International Journal of Human-Computer Interaction, 31(9), 584602.

Leber, M., Ivanišević, A., Borocki, J., Radišić, M., \& Ślusarczyk, B. (2018). Fostering 
alliances with customers for the sustainable product creation. Sustainability, 10(9), 3204.

Leiponen, A., \& Helfat, C. E. (2010). Innovation objectives, knowledge sources, and the benefits of breadth. Strategic management journal, 31(2), 224-236.

Lichtenthaler, U., \& Lichtenthaler, E. (2009). A capability-based framework for open innovation: Complementing absorptive capacity. Journal of management studies, 46(8), 1315-1338.

Maricato, J. D. M. (2010). Dinâmica das relações entre Ciência e Tecnologia: estudo Bibliométrico e Cientométrico de múltiplos indicadores de artigos e patentes em biodiesel (Doctoral dissertation, Universidade de São Paulo).

Nicolaescu, S. S., Palade, H. C., Dumitrascu, D. D., \& Kifor, C. V. (2017). A new project management approach for R\&D software projects in the automotive industrycontinuous V-model. International Journal of Web Engineering and Technology, 12(2), 120-142.

Niemi-Grundström, M. (2014). Developing, evaluating and managing library with agile methods. Library Management.

Noh, H., \& Lee, S. (2015). Perceptual factors affecting the tendency to collaboration in SMEs: Perceived importance of collaboration modes and partners. Journal of technology management \& innovation, 10(3), 18-31.

Nordman, E. R., \& Tolstoy, D. (2016). The impact of opportunity connectedness on innovation in SMEs' foreign-market relationships. Technovation, 57, 47-57.

Otaduy, I., \& Díaz, O. (2017). User acceptance testing for Agile-developed web-based applications: Empowering customers through wikis and mind maps. Journal of Systems and Software, 133, 212-229.

Parida, V., Sjödin, D. R., Lenka, S., \& Wincent, J. (2015). Developing global service innovation capabilities: How global manufacturers address the challenges of market heterogeneity. Research-Technology Management, 58(5), 35-44.

Parida, V., Westerberg, M., \& Frishammar, J. (2012). Inbound open innovation activities in high-tech SMEs: the impact on innovation performance. Journal of small business management, 50(2), 283-309.

Park, E., \& Kwon, S. J. (2018). Effects of innovation types on firm performance: an empirical approach in South Korean manufacturing industry. International Journal of Business Innovation and Research, 15(2), 215-230.

Petticrew, M., \& Roberts, H. (2006). Systematic reviews in the social sciences: A practical guide. Oxford: Blackwell.

Piller, F. T., \& Walcher, D. (2006). Toolkits for idea competitions: a novel method to integrate users in new product development. $R \& d$ Management, 36(3), 307-318.

Prabhu, J. (2016). A survey on slim process with developers in agile software agile software. International Journal of Pharmacy \& Technology, 8(3), 1555815565.

Prahalad, C. K., \& Ramaswamy, V. (2004). Co-creation experiences: The next practice in value creation. Journal of interactive marketing, 18(3), 5-14.

Randhawa, K., Wilden, R., \& Hohberger, J. (2016). A bibliometric review of open innovation: Setting a research agenda. Journal of Product Innovation Management, 33(6), 750-772.

Rusu, G., \& Avasilcăi, S. (2015, November). Innovation management based on proactive engagement of customers: A case study on LEGO Group. Part I: Innovation Management at Lego Group. In IOP Conference Series: Materials Science and Engineering (Vol. 95, p. 012143). 
Ryzhkova, N., \& Pesämaa, O. (2015). Absorptive capacity, collaboration with customers and innovation performance of gazelle companies in knowledge-intensive industries. International Journal of Innovation Management, 19(05), 1550059.

Rose, J., Jones, M., \& Furneaux, B. (2016). An integrated model of innovation drivers for smaller software firms. Information \& Management, 53(3), 307-323.

Shim, W., \& Lee, S. W. (2019). An agile approach for managing requirements change to improve learning and adaptability. Journal of Industrial Information Integration, 14, 16-23.

Schuh, G., Dölle, C., Kantelberg, J., \& Menges, A. (2018). Identification of Agile Mechanisms of Action As Basis for Agile Product Development. Procedia CIRP, 70, 19-24.

Sommer, A. F., Hedegaard, C., DukovskaPopovska, I., \& Steger-Jensen, K. (2015). Improved product development performance through Agile/Stage-Gate hybrids: The nextgeneration Stage-Gate process?. ResearchTechnology Management, 58(1), 34-45.

Silva, G., \& Pereira da Silva, D. E. (2015). Open innovation in services and the role of the client in the business environment: an analysis with university students. Navusrevista de gestao e tecnologia, 5(3), 74-87.

Stefan, I., \& Bengtsson, L. (2017). Unravelling appropriability mechanisms and openness depth effects on firm performance across stages in the innovation process. Technological Forecasting and Social Change, 120, 252-260.

Teece, D. J. (1992). Competition, cooperation, and innovation: Organizational arrangements for regimes of rapid technological progress. Journal of economic behavior \& organization, 18(1), 1-25.

Tether, B. S. (2002). Who co-operates for innovation, and why: an empirical analysis. Research policy, 31(6), 947-967.
Tidd, J., Bessant, J., \& Pavitt, K. (2008). Gestão da inovação. Bookman Editora.

Tidd, J., \& Bessant, J. (2015). Gestão da inovação. Bookman Editora.

Tigre, P. B. (2006). Gestão da inovação. A economia da tecnologia no Brasil. Rio de Janeiro: Editora Campus.

Thanasopon, B., Papadopoulos, T., \& Vidgen, R. (2018). How do firms open up the frontend of service innovation? A case study of IT-based service firms in Thailand. International Journal of Innovation Management, 22(01), 1850010.

Tobiassen, A. E., \& Pettersen, I. B. (2018). Exploring open innovation collaboration between SMEs and larger customers. Baltic Journal of Management.

Tranekjer, T. L. (2017). Open Innovation: effects from external knowledge sources on abandoned innovation projects. Business Process Management Journal, 23(5), 918935.

Tranfield, D., Denyer, D., \& Smart, P. (2003). Towards a methodology for developing evidence-informed management knowledge by means of systematic review. British journal of management, 14(3), 207-222.

Triguero, A., \& Fernández, S. (2018). Determining the effects of open innovation: the role of knowledge and geographical spillovers. Regional Studies, 52(5), 632-644.

Uikey, N., \& Suman, U. (2016). Tailoring for agile methodologies: a framework for sustaining quality and productivity. International Journal of Business Information Systems, 23(4), 432455.

Van de Vrande, V., De Jong, J. P., Vanhaverbeke, W., \& De Rochemont, M. (2009). Open innovation in SMEs: Trends, motives and management challenges. Technovation, 29(6-7), 423-437. 
Silveira, T. B. J., \& Lemos, D. da C. (2021, Ed. Esp. jan./abr.). Colaboração com clientes e inovação aberta no desenvolvimento àgil de software: uma revisão sistemática de literatura

Von Hippel, E. (1988). The Sources of Innovation. Oxford University Press.

Von Hippel, E. (2005). Democratizing innovation: The evolving phenomenon of user innovation. Journal für Betriebswirtschaft, 55(1), 63-78.

Zahra, S. A., \& George, G. (2002). Absorptive capacity: A review, reconceptualization, and extension. Academy of management review, 27(2), 185-203.

Zastempowski, M., \& Przybylska, N. (2016). Cooperation in Creating Innovation in Polish Small and Medium-Sized Enterprises in the Light of Empirical Studies. Journal of Competitiveness, 8(2). 